\title{
BMJ Open Frequency of non-motor symptoms in Parkinson's disease presenting to tertiary care centre in Pakistan: an observational, cross-sectional study
}

To cite: Mukhtar S, Imran R, Zaheer $\mathrm{M}$, et al. Frequency of non-motor symptoms in Parkinson's disease presenting to tertiary care centre in Pakistan: an observational, cross-sectional study. BMJ Open 2018;8:e019172. doi:10.1136/ bmjopen-2017-019172

- Prepublication history for this paper is available online. To view these files, please visit the journal online (http://dx.doi. org/10.1136/bmjopen-2017019172).

Received 2 October 2017 Revised 18 April 2018 Accepted 19 April 2018

Check for updates

${ }^{1}$ Department of Neurology,

Punjab Institute of Neurosciences, Lahore General Hospital, Lahore, Punjab,

Pakistan

${ }^{2}$ School of Biological Sciences, University of the Punjab, Lahore, Punjab, Pakistan

Correspondence to

Dr Shahid Mukhtar;

drshahidmukhtar@hotmail.com

\section{ABSTRACT}

Objective To determine the frequency of non-motor symptoms (NMS) in patients of Parkinson's disease (PD) presenting to a movement disorder clinic at a tertiary care centre in Pakistan, and how frequency of NMS is different in male and female patients.

Study design Observational, cross-sectional study. Setting Tertiary care centre.

Participants Out of 102 patients, 85 were included. Inclusion criteria were patients with PD diagnosed according to the UK Parkinson's Disease Society Brain Bank Clinical Diagnostic Criteria, age $\geq 18$ years, able to give consent and have no difficulty in answering questions. Exclusion criteria were diseases that resemble PD, stroke, dementia, patients unable to provide information and history of antipsychotic use.

Results The NMSQuest revealed a mean of nearly seven different NMS per patient. Autonomic problems such as constipation (56\%) and nocturia (49\%) were the most common NMS, while urinary urgency was reported by $35 \%$ of patients. Low mood and feeling sad were reported by $47 \%$, whereas feeling anxious/panicky was reported by $36 \%$. Problem with memory was reported by $45 \%$ of patients. Feeling of light-headedness and dizziness was reported by $40 \%$ of patients. Problems with sexual relationship were reported by $30 \%$ of patients. The most common sleep problem was difficulty falling sleep $(29 \%)$. Pain not related to the musculoskeletal system was reported by $30 \%$ of patients. Loss or change in the ability to taste or smell was reported by $29 \%$ of patients. The rest of NMS were less than $25 \%$ in frequency. Feeling sad or blue, feeling light-headed/dizzy, unexplained pain, unpleasant sensations in the legs, difficulty in swallowing and faecal incontinence were more common in female participants, while problems with sex were more common in male participants.

Conclusion NMS are quite prevalent in PD in our population. Certain NMS are more common in women as compared with men. There is a need for a large-scale study to look for the association of different NMS with sex.

\section{INTRODUCTION}

Parkinson's disease (PD) is the second most common neurodegenerative disorder, after Alzheimer disease. It is characterised by motor symptoms that include resting tremor,

\section{Strengths and limitations of this study}

- This is the first study on this subject done in Pakistan (of 102 patients, 85 were included in the study) showing 7 non-motor symptoms (NMS) per patient.

- The study compared NMS between male and female patients.

Limitations are its relatively small sample size and that it is a single-centre study.

bradykinesia, rigidity and postural problems. About 1-2 persons per 1000 of the population suffer from PD. The prevalence of PD tends to increase with ageing population, and $1 \%$ of the population above 60 years is affected by PD. ${ }^{2}$ Non-motor symptoms (NMS) of PD include a variety of symptoms such as neuropsychiatric problems (cognitive impairment, anxiety, depression, psychosis, compulsive disorders and apathy), autonomic manifestations (bowel problems such as constipation, urinary complaints and sexual dysfunction), sleep disorders, fatigue and sensory symptoms. ${ }^{3}$

As much as $90 \%$ of people with PD report NMS from early to advanced stages of the disease. ${ }^{4} \mathrm{~A}$ number of studies have shown a negative correlation between NMS and quality of life. ${ }^{5}$

Some NMS of PD are well recognised, such as dementia, dysautonomia, depression and sleep problems, but symptoms such as weight changes, dribbling of saliva, visual problems and sexual problems may not be well appreciated. ${ }^{6}$ Many NMS such as pain, apathy, bowel incontinence, difficulties with sexual relationship and sleep problems may not be disclosed to attending physicians mainly due to embarrassment or patients may be unaware that the symptoms are linked to PD. ${ }^{7} \mathrm{Up}$ to $50 \%$ of patients with PD may have dementia after 15 years of disease period, ${ }^{8}$ with prevalence increasing to as high as $80 \%-90 \%$ by the age 
of 90 years and above. ${ }^{9}$ Neuropsychiatric manifestations, mainly psychosis and depression, are prevalent at all stages of the disease and lead to significant disability. ${ }^{10}$ Many of the drugs that are used for treatment of PD, such as dopamine therapy, can lead to many NMS, such as drug-induced psychosis or hallucinations, postural hypotension and dopamine dysregulation syndrome. ${ }^{11}$ NMS of PD may be very troublesome and the quality of life of both patients with PD and their relatives or caregivers is badly affected. ${ }^{12}$

Most of the times NMS are overlooked during routine patient encounters, and such under-recognition may lead to deleterious implications on the quality of care as many NMS are potentially treatable. ${ }^{7}$ A study conducted in the USA reported that treating physicians in over $50 \%$ of consultations did not recognise existing anxiety, depression, fatigue and sleep problems in over $40 \%$ of encounters. ${ }^{13}$ Recognition of NMS is of utmost importance as many of the NMS of PD are treatable and patients may get better with dopaminergic drugs. ${ }^{10}$

The NMS questionnaire is a comprehensive 30-item self-assessment screening instrument comprising nine NMS domains meant for rapid screening of NMS, empowering both caregiver/relative and patients to raise relevant NMS that are not otherwise discussed in routine clinical visits. ${ }^{14}$

The validity, feasibility and acceptability of the NMS questionnaire have been assessed in a pilot validation study, which has been published, and the questionnaire has undergone independent validation and is hence recommended by the Department of Health in the UK for use in clinical practice. ${ }^{7}$

There have been many studies conducted to date that described different NMS in different populations, but very few studies were conducted in this part of the world. The main idea that motivated this research project was to see the pattern of NMS in our population and see if there is any difference between male and female patients in regard to the frequency of the different NMS.

\section{PATIENTS AND METHODS}

This was a cross-sectional, observational and descriptive study. It was conducted on patients with PD presenting to a movement disorder clinic, Lahore General Hospital, Lahore (a tertiary care centre) from 1 August 2015 to 31 December 2016. The inclusion criteria were patients with PD diagnosed according to the UK Parkinson's Disease Society Brain Bank Clinical Diagnostic Criteria, ${ }^{15}$ age $\geq 18$ years, able to give consent, and have no difficulty in answering questions independently or with the help of a family member or a doctor. The exclusion criteria were diseases that resemble PD (Parkinson's plus others), patients with stroke, patients who were unable to provide information, dementia and history of antipsychotic use. Seventeen patients were found to have Parkinson's plus, concurrent stroke and history of taking antipsychotics, and hence were not included in the study. Informed consent was obtained. All participants of the study were required to fill in a PD NMS questionnaire either himself or with the help of a family member. In cases where there was a language problem, doctors helped patients in filling in the PD NMS questionnaire. Responses were recorded in a yes or no fashion. Patients only marked yes if he or she had experienced the said symptom/problem in the past 1 month. Drug history and routine demographic details were also recorded. Declaration of NMS by patients with PD led to proper management of reported symptoms.

G*Power V.3.1 statistical software was used for sample size determination. By using one-sample t-test, 'NMS' score was assumed as an outcome variable. The mean and SD of NMS were considered at $35.21 \pm 21.85 .^{16}$ The calculated effect size and power (1- $\beta$ ) were 0.80 and 0.95 , respectively. An aggregate of 85 samples would be quite enough for our study parameters.

\section{Patient and public involvement statement}

No patient was involved in the design or conduct of the study, but the results of the study will be shared to patients coming for follow-up.

\section{Data analysis}

After the questionnaires were filled in by all of the patients, data were entered and analysed using Statistical Package for Social Sciences (SPSS) V.22. The mean and SD were calculated for numerical variables. Categorical data were expressed as frequency and percentage. With regard to the reliability of NMS among items, a Cronbach's alpha value of 0.72 reflected good reliability. The frequency of each NMS was calculated by computing the number of yes response and calculating the percentage related to the number of patients in the sample. Analysis was done to calculate the frequency of all NMS among the enrolled patients. To find out the association of NMS symptoms with respect to male and female patients, $\chi^{2}$ test/Fisher's exact test was applied and $\mathrm{p} \leq 0.05$ was considered significant.

\section{RESULTS}

From August 2015 to 31 December 2016, 102 patients were screened. Out of those patients, 85 were found eligible for study based on the inclusion and exclusion criteria. The average age was $57.61 \pm 10.64$ years, the youngest being 35 years old and the oldest being 77 years old. In our study, men dominated in number $(\mathrm{n}=70,82 \%)$ as compared with women $(n=15,18 \%)$. The NMS score revealed a mean of nearly seven different NMS per patient. Gastrointestinal complaints were the most frequent. Out of a total of 85 patients, $48(56 \%)$ reported constipation. Only 24 (28\%) patients reported troublesome sialorrhoea. Urinary symptoms were next in frequency, with nocturia being the second most common symptom as reported by $42(49 \%)$ patients, while urinary urgency was reported by $30(35 \%)$ patients. Neuropsychiatric symptoms were also quite frequent as low mood and feeling 
sad were reported by $40(47 \%)$ patients, whereas feeling anxious and panicky was reported by $31(36 \%)$. Problems with memory were reported by $38(45 \%)$ patients. Problems with libido were reported by $12(14 \%)$ patients and $26(30 \%)$ patients admitted difficulty with sexual relationships. Among autonomic symptoms, feeling of light-headedness and dizziness while standing from lying or sitting position was reported by $35(40 \%)$ patients. Difficulty falling sleep and staying asleep was reported by 25 (29\%) patients. Pain not related to the musculoskeletal system was reported by $26(30 \%)$ patients. Change in the ability to taste or smell was reported by $25(29 \%)$ patients. Excessive sweating, unpleasant sensation in the legs, frightening dreams and unexplained change in weight were reported by $21(24 \%), 19(22 \%), 19(22 \%)$ and $18(20 \%)$ patients, respectively. The rest of the NMS were less than $20 \%$ in frequency. The frequency of all NMS is listed in table 1 .

The most common NMS in men included constipation $(54 \%)$, getting up regularly to pass urine at night $(50 \%)$ and problems with short-term memory (48\%). The most common NMS in women included feeling sad or blue (80\%), unexplained pain (67), feeling light-headed or dizzy (73\%), feeling anxious or panicky (61\%), and feeling unpleasant sensations in the legs while at rest $(47 \%)$. The frequency of all NMS in both sexes is shown in figure 1.

We observed that a significant association of NMS symptoms was found as compared with men and women in feeling sad or blue, feeling light-headed/dizzy, unexplained pain, problems with sex, unpleasant sensation in the legs, difficulty in swallowing food or drink or problems with choking, and faecal incontinence (table 2). However the small number of female patients $(n=15)$ was a major limitation of our study.

\section{DISCUSSION}

In this study we have shown the high frequency of NMS in patients with PD in Pakistan. Overall the most frequent NMS was constipation (56\%). In our clinical practice, constipation is in fact a very common complaint. Stocchi and Torti $^{17}$ described the prevalence of constipation in PD ranging from $24.6 \%$ to $63 \%$ depending on different diagnostic criteria. In PD it is due to slow colonic transit and it can be relieved by polyethylene glycol and lubiprostone, along with dietary modifications. ${ }^{18}$ Nocturia is second in frequency $(49 \%)$, while urinary urgency was reported by $35 \%$ of patients. Chaudhuri et $a l^{7}$ reported nocturia to be the most common NMS (65\%) in their study. Weerkamp et $a l^{19}$ described nocturia in $57 \%$ of nursing home residents. Yeo $e t a l^{20}$ described nocturia as the most common urinary tract complaint in patients with PD $(>60 \%)$ and attributed it to detrusor muscle overactivity, while urgency was found at $33 \%-54 \%$. Rana $e t a l^{21}$ reported a significant association between anxiety and nocturia in patients with PD, with greater significance found for male patients as compared with female patients, and suggested that addressing anxiety may help nocturia.
Table 1 Prevalence of individual non-motor symptoms (NMS) items

\begin{tabular}{|c|c|}
\hline NMS symptoms & $\begin{array}{l}\text { Percentage } \\
\text { (frequency) }\end{array}$ \\
\hline Constipation & $56(48)$ \\
\hline Nocturia & $49(42)$ \\
\hline Feeling sad or blue & $47(40)$ \\
\hline Forgetting to do things & $45(38)$ \\
\hline Feeling light-headed/dizzy & $40(35)$ \\
\hline Feeling anxious or panicky & $36(31)$ \\
\hline Urinary urgency & $35(30)$ \\
\hline Unexplained pain & $30(26)$ \\
\hline Problems with sex & $30(26)$ \\
\hline Difficulty sleeping & $29(25)$ \\
\hline $\begin{array}{l}\text { Loss or change in ability to taste or } \\
\text { smell }\end{array}$ & $29(25)$ \\
\hline Loss of interest in surroundings & $29(25)$ \\
\hline Dribbling of saliva & $28(24)$ \\
\hline Excessive sweating & $24(21)$ \\
\hline Unpleasant sensation in the legs & 22 (19) \\
\hline Frightening dreams & 22 (19) \\
\hline Unexplained change in weight & $20(18)$ \\
\hline Falling & $19(15)$ \\
\hline $\begin{array}{l}\text { Difficulty in swallowing food or drink } \\
\text { or problems with choking }\end{array}$ & $17(15)$ \\
\hline $\begin{array}{l}\text { Difficulty concentrating or staying } \\
\text { focused }\end{array}$ & $16(14)$ \\
\hline Swelling in the legs & $14(12)$ \\
\hline Daytime somnolence & $13(11)$ \\
\hline Double vision & $14(12)$ \\
\hline Decreased/increased libido & $14(12)$ \\
\hline Talking or moving in sleep & $11(10)$ \\
\hline Incomplete bowel emptying & $11(10)$ \\
\hline Vomiting or feeling of sickness & $10(9)$ \\
\hline Delusions & $8(7)$ \\
\hline Illusions & $8(7)$ \\
\hline Faecal incontinence & $6(5)$ \\
\hline
\end{tabular}

Neuropsychiatric symptoms such as anxiety, low mood and memory problems can lead to significant morbidity and negatively impact quality of life. Feeling anxious was reported by $36 \%$ of our patients, while $47 \%$ had low mood and $45 \%$ had issues with remembering things. Chaudhuri et al and Weerkamp et al reported anxiety in $42 \%$ and $53 \%$ and low mood in $49 \%$ and $66 \%$, respectively, while $51 \%$ of patients had problems with their memory in both studies. ${ }^{719}$ These symptoms in PD are attributable to intricate pathological changes in various brain regions. ${ }^{22}$ Alterations in dopamine, acetylcholine and serotonin levels in subcortical projections and degenerative processes involving the limbic circuits and 


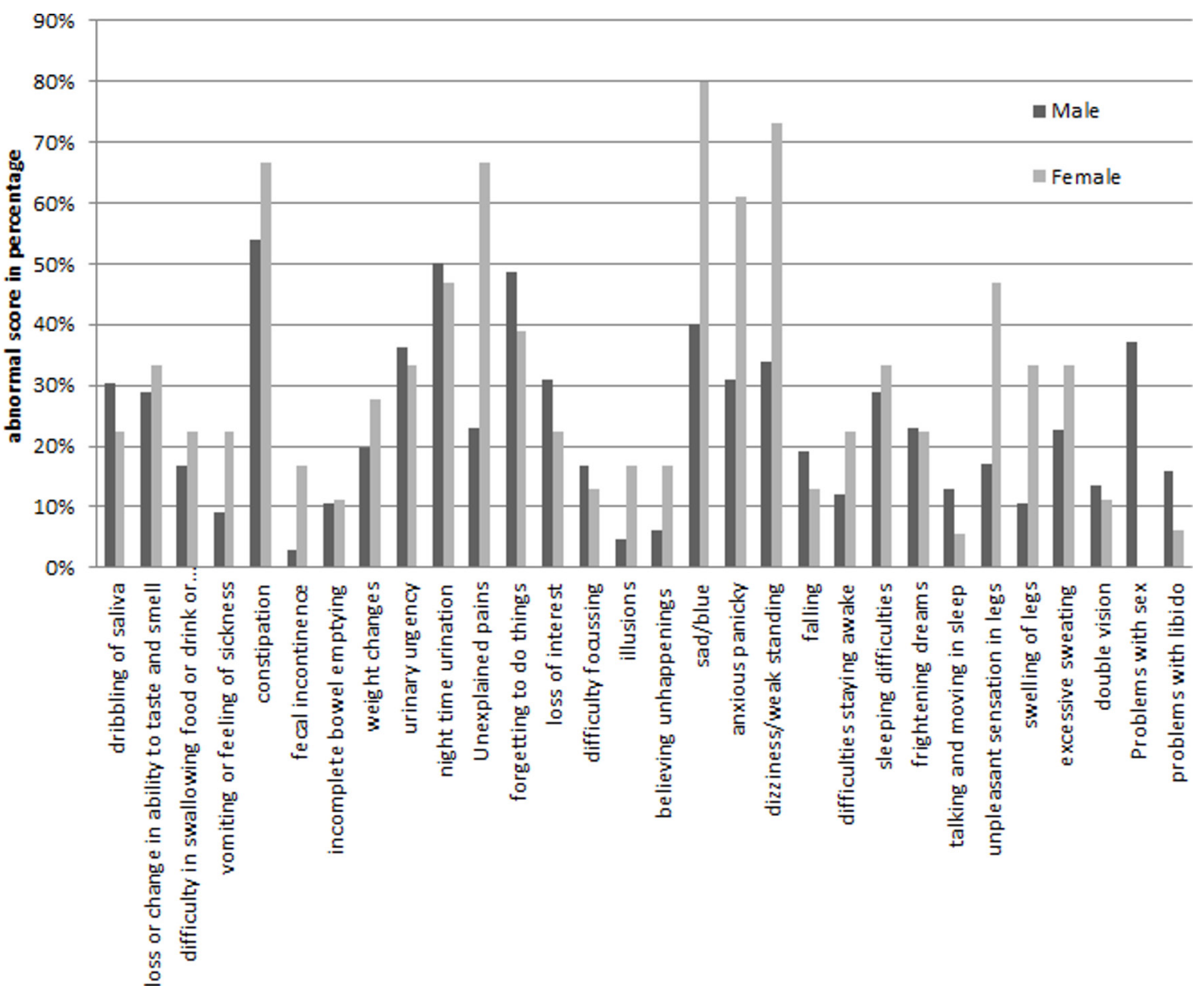

Figure 1 Frequency of different non-motor symptoms (NMS) in both sexes. The $x$-axis shows different NMS while the $y$-axis shows the percentage.

cortical connections lead to NMS. ${ }^{23}$ Tricyclic antidepressants are effective for depression in PD, and antipsychotic clozapine is effective for psychosis. ${ }^{24}$

Feeling light-headed and dizzy was also a major concern (40\%) in our study. Other studies have reported slightly higher incidences $(39 \%$ and $48 \%){ }^{719}$ It may be relieved by addressing neurogenic orthostatic hypotension. Management includes patient education, physical manoeuvres, adequate hydration and judicious use of dopaminergic drugs. ${ }^{25}$ Specific treatment includes salt supplementation, mineralocorticoids and other empiric medications. ${ }^{26}$

Drooling of saliva is one of the NMS in patients with PD that have negative physical and psychosocial impact on patients and their families. The cause of drooling in PD remains uncertain; however, decreased intraoral salivary clearance is thought to be the major factor in the pathophysiology of drooling. ${ }^{26}$ Our study shows that $28 \%$ of patients had drooling of saliva, while other studies found a higher number of patients with this complaint $(42 \%$ and $63 \%) .^{719}$ This difference may be due to the difference in age of patients (our cohort being younger) and also the frequent use of anticholinergics in this part of the world. Non-pharmacological measures such as sucking on candies or chewing gum ${ }^{27}$ and adjusting dopaminergic drugs may help some patients. ${ }^{28}$ Drug therapy for severe drooling includes muscarinic receptor antagonist, which inhibits salivary secretions. However, keeping in mind their adverse effect profiles, such agents should be used cautiously in elderly patients. ${ }^{29}$ Injection of parotid glands with botulinum toxin has been shown to be a safe and effective modality in the treatment of sialorrhoea in patients with PD. ${ }^{30-33}$

Sleep problems in patients with PD comprise problems with initiating sleep, frequent night-time awakening, restless legs syndrome, sleep breathing disorders, rapid eye movement sleep disorders, sleep attacks, drug-induced symptoms and daytime somnolence. ${ }^{34}$ In our study $29 \%$ of patients had problems with insomnia, $14 \%$ had daytime somnolence and $10 \%$ had problem with talking or moving in sleep. Treatment of insomnia includes ruling out and treating other sleep-related motor and breathing problems, treating motor complications of PD if present, following proper sleep hygiene and cognitive-behavioural therapy, and melatonin at high dose, and if all ineffective then drugs such as zolpidem, trazodone or doxepin, and eszopiclone could be added. ${ }^{35}$

Anosmia is a common NMS of PD. Anosmia has been shown to occur years before the motor symptoms of PD set in, and autopsy findings have confirmed synucleinopathy 
Table 2 Association of non-motor symptoms (NMS) with sex

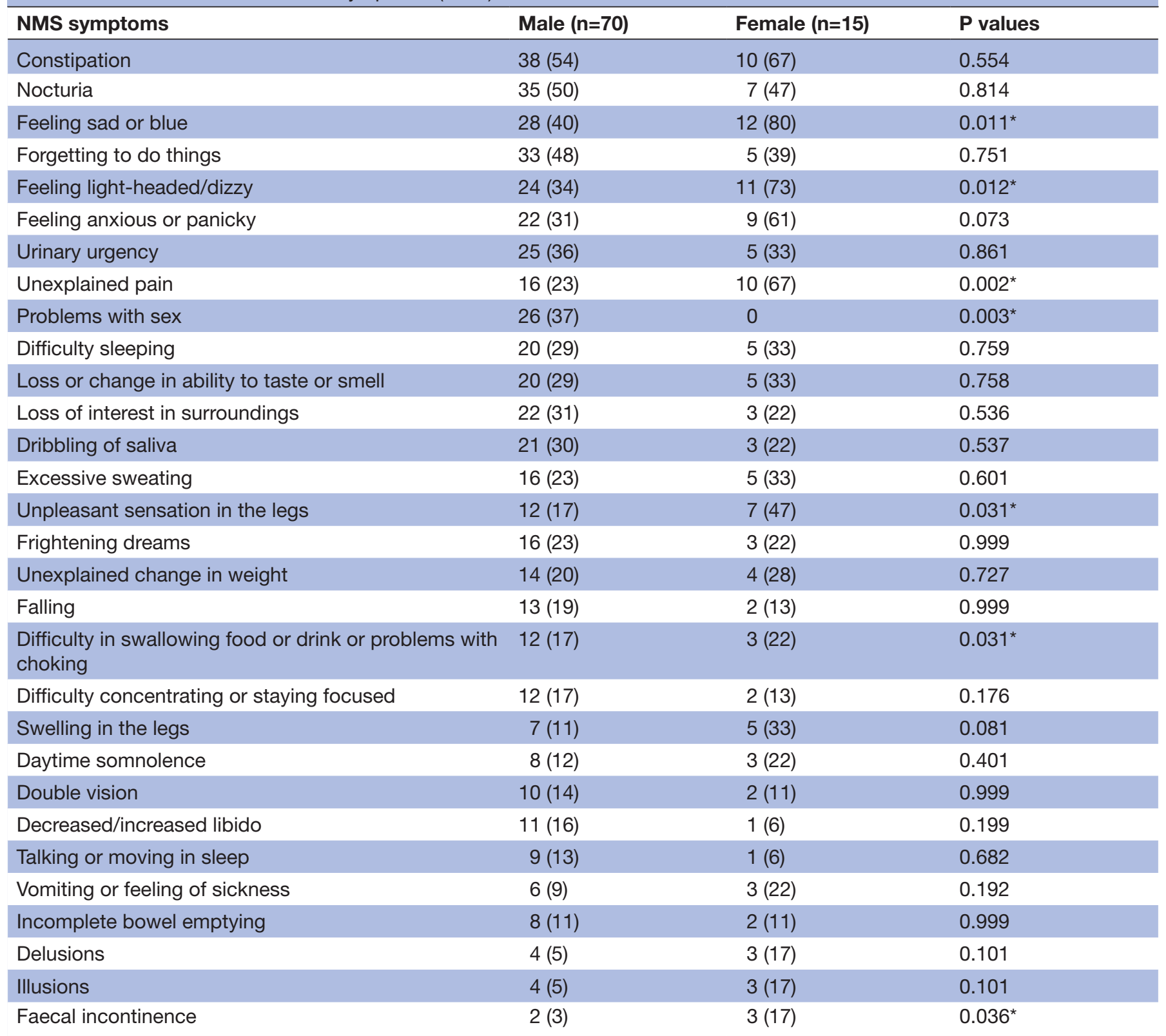

Categorical variables were presented as frequencies (percentages) and $\chi^{2} /$ Fisher's exact test was applied.

*Significant $(p \leq 0.05)$.

affecting the olfactory pathways and the anterior olfactory nucleus. ${ }^{36}$ In our study $29 \%$ of patients reported anosmia and ageusia. Other studies have reported different frequencies $(43 \%$ and $11 \%) .{ }^{719}$ The difference in reported frequencies may be due to different cultures, age of patients, disease stage and different sample sizes.

Sexual dysfunction is an important but unfortunately a neglected NMS of PD. In our study $13 \%$ of patients reported problems with libido, while $30 \%$ voiced problems with sexual intercourse. Chaudhuri et $a l^{7}$ reported problems with libido in $37 \%$ of their patients and $34 \%$ of their patients had difficulty with sex. Kummer et $a l^{37}$ reported loss of libido in $65.6 \%$ of their patients, and showed advancing age, lower education, female sex and depression were associated with low libido. The underlying pathology is hypothalamic dysfunction via altered dopamine-oxytocin pathways, which normally promote libido. ${ }^{38}$ Varanda $e t a l^{39}$ described sexual dysfunction in women with PD and reported it at $86.9 \%$. Interestingly $\mathrm{Hu}$ et at ${ }^{40}$ conducted a study on Chinese male patients and reported sexual dysfunction was found in $41.4 \%$ of their patients. A possible explanation of these differences could be due to different cultures, social values and possible biases in reporting. This topic should be addressed with adequate privacy. Also social norms should be given due respect while looking into these problems.

Certain NMS were more common in female patients $(\mathrm{p} \leq 0.05)$. They include feeling sad or blue, feeling lightheaded/dizzy, unexplained pain, unpleasant sensation in the legs, difficulty in swallowing food or drink or problems 
with choking, and faecal incontinence. This implies that female patients need to be probed more thoroughly for NMS. Problems with sex were the only NMS that was more frequent in male patients $(p \leq 0.05)$. This may be due to reporting bias. Women in Pakistani culture are more conservative and usually do not feel comfortable with discussing such intimate issues.

Our study had some limitations as well. Majority of our patients were male $(\mathrm{n}=70,82 \%)$ as compared with female $(\mathrm{n}=15,18 \%)$, with a male to female ratio of $4.6: 1$. This may be due to culture bias, as expected in male-dominant societies where men are more likely to seek medical care. The study group was relatively small in number and was performed at one centre, so the study may have an element of selection bias as well (investigating only patients coming to our movement disorder clinic).

\section{CONCLUSION}

NMS are quite prevalent in PD in our population. Certain NMS are more common in female patients as compared with male patients. There is a need for a large-scale study to look for an association of different NMS with sex. This will lead to better understanding of patients' problems and more vigilant care can hence be provided.

Acknowledgements Special thanks to Mr. IIfan Majeed for his continuous support.

Contributors SM: designed the study and looked after the whole project. RI: worked with the patients, applied the questionnaire, helped in data collection. MZ: supervised the study, helped with ambiguities, analysis and interpretation. HT: applied the questionnaire, helped in data collection.

Funding The authors have not declared a specific grant for this research from any funding agency in the public, commercial or not-for-profit sectors.

Competing interests None declared.

Patient consent Obtained.

Ethics approval Hospital ethical committee for clinical disciplines, Lahore General Hospital.

Provenance and peer review Not commissioned; externally peer reviewed.

Data sharing statement Data can be shared via email with interested readers.

Open Access This is an Open Access article distributed in accordance with the Creative Commons Attribution Non Commercial (CC BY-NC 4.0) license, which permits others to distribute, remix, adapt, build upon this work non-commercially, and license their derivative works on different terms, provided the original work is properly cited and the use is non-commercial. See: http://creativecommons.org/ licenses/by-nc/4.0/

(C) Article author(s) (or their employer(s) unless otherwise stated in the text of the article) 2018. All rights reserved. No commercial use is permitted unless otherwise expressly granted.

\section{REFERENCES}

1. Chaudhuri KR, Sauerbier A, Rojo JM, et al. The burden of non-motor symptoms in Parkinson's disease using a self-completed non-motor questionnaire: a simple grading system. Parkinsonism Relat Disord 2015;21:287-91.

2. Tysnes OB, Storstein A. Epidemiology of Parkinson's disease. J Neural Transm 2017;124:901-5.

3. Bostantjopoulou S, Katsarou Z, Karakasis C, et al. Evaluation of non-motor symptoms in Parkinson's Disease: An underestimated necessity. Hippokratia 2013;17:214-9.

4. Khoo TK, Yarnall AJ, Duncan GW, et al. The spectrum of nonmotor symptoms in early Parkinson disease. Neurology 2013;80:276-81.
5. Barone P, Antonini A, Colosimo C, et al. The PRIAMO study: A multicenter assessment of nonmotor symptoms and their impact on quality of life in Parkinson's disease. Mov Disord 2009;24:1641-9.

6. Chaudhuri KR, Naidu Y. Early Parkinson's disease and non-motor issues. J Neurol 2008;255(Suppl 5):33-8.

7. Chaudhuri KR, Prieto-Jurcynska C, Naidu Y, et al. The nondeclaration of nonmotor symptoms of Parkinson's disease to health care professionals: An international study using the nonmotor symptoms questionnaire. Movement Disorders 2010;25:704-9.

8. Hely MA, Morris JG, Reid WG, et al. Sydney multicenter study of parkinson's disease: non-I-dopa-responsive problems dominate at 15 years. Mov Disord 2005;20:190-9.

9. Buter TC, van den Hout A, Matthews FE, et al. Dementia and survival in Parkinson disease: a 12-year population study. Neurology 2008;70:1017-22.

10. Chaudhuri KR, Schapira AH. Non-motor symptoms of Parkinson's disease: dopaminergic pathophysiology and treatment. Lancet Neurol 2009:8:464-74.

11. Chaudhuri KR, Healy DG, Schapira AH. National Institute for Clinical Excellence. Non-motor symptoms of Parkinson's disease: diagnosis and management. Lancet Neurol 2006;5:235-45.

12. Sauerbier A, Chaudhuri KR. Non-motor symptoms: the core of multimorbid Parkinson's disease. Br J Hosp Med 2014;75:18-24.

13. Shulman LM, Taback RL, Rabinstein AA, et al. Non-recognition of depression and other non-motor symptoms in Parkinson's disease. Parkinsonism Relat Disord 2002;8:193-7.

14. Chaudhuri KR, Martinez-Martin P, Schapira AH, et al. International multicenter pilot study of the first comprehensive self-completed nonmotor symptoms questionnaire for Parkinson's disease: the NMSQuest study. Mov Disord 2006;21:916-23.

15. Hughes AJ, Daniel SE, Kilford L, et al. Accuracy of clinical diagnosis of idiopathic Parkinson's disease: a clinico-pathological study of 100 cases. J Neurol Neurosurg Psychiatry 1992;55:181-4.

16. Ravan A, Ahmad FM, Chabria S, et al. Non-motor symptoms in an Indian cohort of Parkinson's disease patients and correlation of progression of non-motor symptoms with motor worsening. Neurol India 2015;63:166-74.

17. Stocchi F, Torti M. Constipation in Parkinson's disease. Int Rev Neurobiol 2017;134:811-26.

18. Rossi M, Merello M, Perez-Lloret S. Management of constipation in Parkinson's disease. Expert Opin Pharmacother 2015;16:547-57.

19. Weerkamp NJ, Tissingh G, Poels PJ, et al. Nonmotor symptoms in nursing home residents with Parkinson's disease: prevalence and effect on quality of life. J Am Geriatr Soc 2013;61:1714-21.

20. Yeo L, Singh R, Gundeti M, et al. Urinary tract dysfunction in Parkinson's disease: a review. Int Urol Nephrol 2012;44:415-24.

21. Rana AQ, Paul DA, Qureshi AM, et al. Association between nocturia and anxiety in Parkinson's disease. Neurol Res 2015;37:563-7.

22. Szatmari S, Illigens BM, Siepmann T, et al. Neuropsychiatric symptoms in untreated Parkinson's disease. Neuropsychiatr Dis Treat 2017;13:815-26.

23. Oikonomou E, Paparrigopoulos T. [Neuropsychiatric manifestations in Parkinson's disease]. Psychiatriki 2015;26:116-30.

24. Aarsland D, Kramberger MG. Neuropsychiatric symptoms in Parkinson's disease. J Parkinsons Dis 2015;5:659-67.

25. Isaacson SH, Skettini J. Neurogenic orthostatic hypotension in Parkinson's disease: evaluation, management, and emerging role of droxidopa. Vasc Health Risk Manag 2014;10:169-76.

26. Srivanitchapoom P, Pandey S, Hallett M. Drooling in Parkinson's disease: a review. Parkinsonism Relat Disord 2014;20:1109-18.

27. Trpcje MS, Fernandex HH. Sialorrhea and Parkinson disease: novel treatment approach. Neurology 2010;6:423-4.

28. Hockstein NG, Samadi DS, Gendron K, et al. Sialorrhea: a management challenge. Am Fam Physician 2004;69:2628-34.

29. Nishtala PS, Fois RA, McLachlan AJ, et al. Anticholinergic activity of commonly prescribed medications and neuropsychiatric adverse events in older people. J Clin Pharmacol 2009;49:1176-84.

30. Gómez-Caravaca MT, Cáceres-Redondo MT, Huertas-Fernández I, et al. The use of botulinum toxin in the treatment of sialorrhea in parkinsonian disorders. Neurol Sci 2015;36:275-9.

31. Lagalla G, Millevolte M, Capecci M, et al. Botulinum toxin type A for drooling in Parkinson's disease: a double-blind, randomized, placebo-controlled study. Mov Disord 2006;21:704-7.

32. Lagalla G, Millevolte M, Capecci M, et al. Long-lasting benefits of botulinum toxin type B in Parkinson's disease-related drooling. $J$ Neurol 2009;256:563-7.

33. Kalf JG, Smit AM, Bloem BR, et al. Botulinum toxin A for drooling in Parkinson's disease: a pilot study to compare submandibular to parotid gland injections. Parkinsonism Relat Disord 2007;13:532-4. 
34. Raggi A, Bella R, Pennisi G, et al. Sleep disorders in Parkinson's disease: a narrative review of the literature. Rev Neurosci 2013;24:279-91.

35. Loddo G, Calandra-Buonaura G, Sambati L, et al. The treatment of sleep disorders in parkinson's disease: from research to clinical practice. Front Neurol 2017;8:42.

36. Tarakad A, Jankovic J. Anosmia and ageusia in parkinson's disease. Int Rev Neurobiol 2017;133:541-56.
37. Kummer A, Cardoso F, Teixeira AL. Loss of libido in Parkinson's disease. J Sex Med 2009;6:1024-31.

38. Sakakibara R, Uchiyama T, Yamanishi T, et al. Genitourinary dysfunction in Parkinson's disease. Mov Disord 2010;25:2-12.

39. Varanda S, Ribeiro da Silva J, Costa AS, et al. Sexual dysfunction in women with Parkinson's disease. Mov Disord 2016;31:1685-93.

40. Hu X, Liu WG, Yan FL. [Male sexual dysfunction and Parkinson's disease: a preliminary investigation]. Zhonghua Nan Ke Xue 2013;19:518-21. 